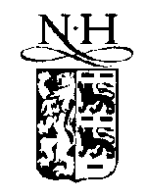

ELSEVIER

\title{
Complex permeability of soft magnetic ferrite/polyester resin composites at frequencies above $1 \mathrm{MHz}$
}

\author{
J.H. Paterson*, R. Devine, A.D.R. Phelps \\ Department of Physics and Applied Physics, University of Strathclyde. John Anderson Building, Glasgow G4 ONG. LK
}

\begin{abstract}
Composite soft magnetic materials consist of magnetic particles in a non-magnetic matrix. The properties of such materials can be modelled using effective medium theory. Measurements have been made of the complex permeability of compositcs produced using ferrite powder and polyester resin. The success of various effective medium expressions in predicting the variation of complex permeability with composition has been assessed. 1999 Elsevier Science B.V. All rights reserved.
\end{abstract}

Kennords: Composites; Ferrites; Effective medium theory; Permeability

Soft magnetic composite materials, produced by embedding soft magnetic particles in a non-magnetic matrix, have a significant advantage over metallic or ceramic materials which can be produced using the established polymer processing techniques. They can be readily moulded into complex shapes to produce magnetic components for specific applications. The relationship between the properties of a composite material and those of its separate components can be modelled using effective medium theory. In this work, the high-frequency complex permeability of a series of ferrite/resin composites has been measured. The results have been compared with the predictions of various formalisms of effective medium theory in order to determine how successful this theory is in modelling the behaviour of these materials over the whole range of possible compositions.

The general effective medium expression for permeability for the case of two components is based on the assumption that the components are embedded in a host medium. The resultant properties are those of the effec-

\footnotetext{
* Corresponding author. Fax: + 44141552 2891; e-mail: j.h.paterson@strath.ac.uk.
}

ive medium. The expression is

$$
\frac{\mu-\mu_{\mathrm{h}}}{\mu+2 \mu_{\mathrm{h}}}=f_{\mathrm{a}}\left(\frac{\mu_{\mathrm{a}}-\mu_{\mathrm{h}}}{\mu_{\mathrm{a}}+2 \mu_{\mathrm{h}}}\right)+f_{\mathrm{b}}\left(\frac{\mu_{\mathrm{h}}-\mu_{\mathrm{h}}}{\mu_{\mathrm{b}}+2 \mu_{\mathrm{h}}}\right) .
$$

where $\mu$ is the permeability of the effective medium, $\mu_{\mathrm{a}}, \iota_{\mathrm{b}}$ and $\mu_{\mathrm{h}}$ are the permeabilities of the two components $\mathrm{a}$ and $\mathrm{b}$ and of the host, respectively, and $f_{\mathrm{a}}$ and $f_{\mathrm{b}}$ are the volume fractions of the components. Some assumptions must be made about the component distribution in the composite, and about the host material. 4 number of different ways of doing this have been proposed $[1,2]$. If the assumption is made that the material consists of isolated particles of component a embedded in component $b$, which is therefore the host. Fq. (1) simplifies to

$\frac{\mu-\mu_{\mathrm{h}}}{\mu+2 \mu_{\mathrm{h}}}=f_{\mathrm{a}}\left(\frac{\mu_{\mathrm{a}}-\mu_{\mathrm{h}}}{\mu_{\mathrm{a}}+2 \mu_{\mathrm{h}}}\right)$.

This is Maxwell-Garnett (MGa) effective medium expression. There is an equivalent expression, MGb, with $b$ as the embedded component. The result for a given composition will be different depending on which of these expression is used. The Bruggeman formalism assumes that both components are embedded in the effective 


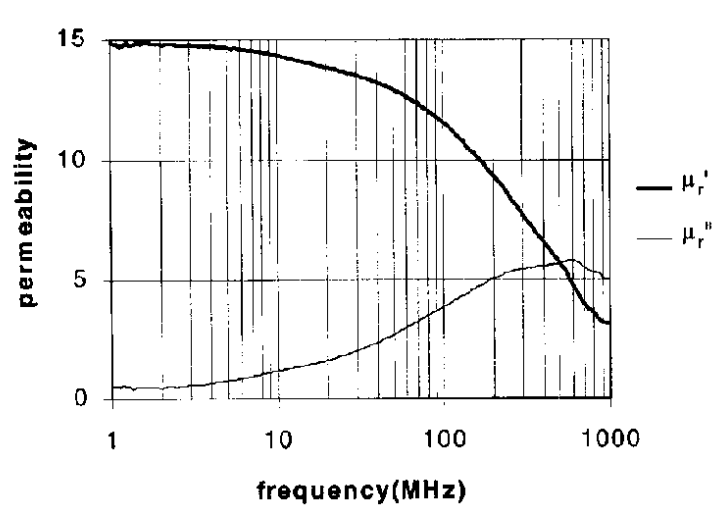

Fig. 1. Typical complex permeability spectrum of ferrite/resin composite.

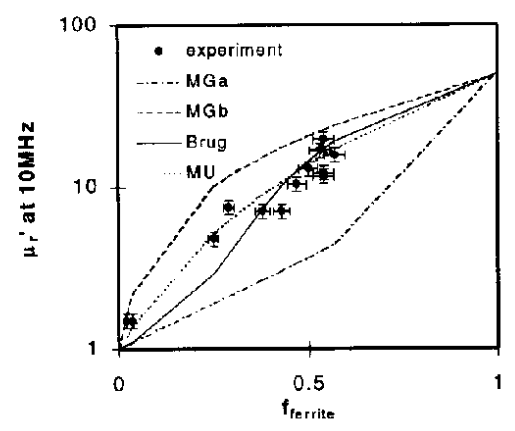

Fig. 2. Experimental and modelled $\mu^{\prime}$ vs. ferrite volume fraction $f_{\text {ferrite }}$ at $10 \mathrm{MHz}$.

medium itself, and is expressed as

$0=f_{\mathrm{a}}\left(\frac{\mu_{\mathrm{a}}-\mu}{\mu_{\mathrm{a}}+2 \mu}\right)+f_{\mathrm{b}}\left(\frac{\mu_{\mathrm{b}}-\mu}{\mu_{\mathrm{b}}+2 \mu}\right)$.

Following the approach of Musal et. al. [2] the Maxwell-Garnett expressions can be extended to work throughout the entire range of compositions $0 \leqslant f_{\mathrm{a}} \leqslant 1$. MGa is useful when $f_{\mathrm{a}}$ is small, since such a composite will appear to consist of isolated particles of a embedded in b. Similarly, MGb is appropriate when $f_{\mathrm{a}}$ is large, and hence $f_{\mathrm{b}}$ is small. Here, the medium is described as a combination of two phases. The isolated phase consists of particles of a embedded in $\mathrm{b}$, while in the agglomerated phase, $\mathrm{a}$ is surrounded by isolated particles of $\mathrm{b}$. The permeability of each phase can be calculated using the appropriate Maxwell-Garnett expression. As $f_{\mathrm{a}}$ increases, the relative concentration of the agglomerated phase will increase. The two phases are combined using a further Maxwell-Garnett expression, corresponding to the agglomerated phase embedded in the isolated phase, with the relative concentrations of the phases dependent on

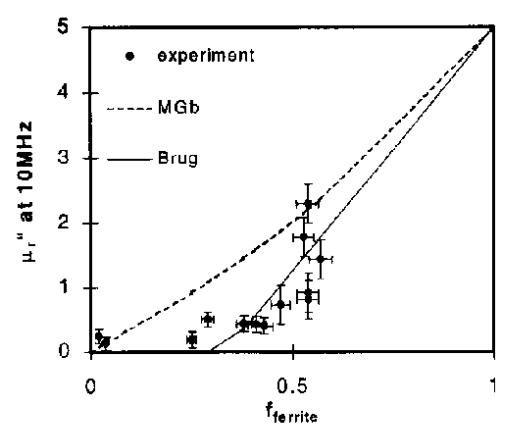

Fig. 3. Experimental and modelled $\mu^{\prime \prime}$ vs. ferrite volume fraction $f_{\text {ferrite }}$ at $10 \mathrm{MHz}$.

$f_{\mathrm{a}}$ and $f_{\mathrm{b}}$. The concentration of the agglomerated phase is defined as $f_{\mathrm{a}}^{x}$, where $x$ is a fitting parameter.

The base magnetic material used in this work was an unsintered manganese-zinc ferrite powder, grade F9C, supplied by MMG-Neosid. The powder had an average particle size $<300 \mu \mathrm{m}$. In its sintered form this material has a nominal initial permeability $\mu_{\mathrm{r}}$ of $\sim 5000$ and has a frequency response suitable for applications below $100 \mathrm{kHz}$. The matrix was a polyester laminating resin. A simple mixing and moulding process was used to produce toroidal composite samples with ferrite volume fractions up to $\sim 50 \%$. The complex permeability of each toroidal sample was measured over the range $1 \mathrm{MHz}-1 \mathrm{GHz}$ using a Hewlett-Packard HP4291B Material Analyser. A typical complex permeability spectrum is shown in Fig. 1. The frequency response is significantly different from that of the sintered material. Figs. 2 and 3 show the experimental measurements of $\mu^{\prime}$ and $\mu^{\prime \prime}$, respectively, vs. ferrite volume fraction at $10 \mathrm{MHz}$, together with the predictions of the effective medium expressions detailed above. The trends in the data were similar at other frequencies in the measured range. The uncertainty in the permeability values was estimated from the manufacturer's specifications for the HP4291B. Since the permeability of the ferrite powder was not accurately known, the values of $\mu_{\text {ferrite }}$ and $\mu_{\text {ferrite }}^{\prime \prime}$ used in the models were adjusted to give the best overall fit to the data. Since the resin is non-magnetic, $\mu_{\text {resin }}^{\prime}=1$ and $\mu^{\prime \prime}{ }_{\text {resin }}=0$. MGa and $\mathrm{MGb}$ give very different results for $\mu^{\prime}$. Neither gives a good fit over the whole range of compositions. The Bruggeman expression (denoted BRUG) is a significant improvement. The Musal approach (denoted MU) was used for $\mu$ ' with the parameter $x=0.33$, chosen empirically to give the best fit. This gives a reasonable fit to all the data. For $\mu^{\prime \prime}, \mathrm{MGa}$ and MU cannot be calculated since the MGa expression gives an undefined result if $\mu_{\mathbf{b}}=0$. BRUG gives a reasonable fit to the data provided $f_{\mathrm{a}}>\frac{1}{3}$, but gives zero below this value. These comparisons suggest that for these materials the Bruggeman expression works reasonably well, 
particularly when the components are present in roughly equal fractions. The Musal approach works well over a wider rangc of composition, but it cannot be used for $\mu^{\prime \prime}$.

\section{Acknowledgements}

This work has been supported by DERA Malvern, UK. The ferrite powder was kindly supplied by Dr. Ian Wilkinson of MMG Neosid.

\section{References}

[1] D.E. Aspnes. Am. J. Phys. 50 (1982) 704

[2] H.M. Musal Jr., H.T. Hahr, G.G. Bush, J. Appl. Phys. 63 (1988) 3768 